\title{
Self-similarity of fluid flows
}

\author{
Liqiu Wanga) \\ Department of Mechanical Engineering, University of Hong Kong, Hong Kong
}

(Received 16 April 1998; accepted for publication 8 July 1998)

Relations among flows at different scales are developed through examining flow response to multiplicative changes of spatial and temporal scales. The scaling invariance of physical definitions leads to the self-similarity of flows and the scaling invariance of fundamental laws governing the flows. Also developed is both the necessary and sufficient condition for a physical quantity to be self-similar, which forms a general function dependence of any self-similar quantity on position and time. (C) 1998 American Institute of Physics. [S0003-6951(98)01436-3]

Fluid flows often involve a wide spectrum of spatial and temporal scales. Typical examples are turbulent, atmospheric and oceanic flows. Such flows cannot be characterized by a single length and time scale. The nonlinear interaction among flows at different scales usually leads to a complex flow structure such as turbulence and climate. Relations among flows at different scales are, thus, of crucial importance in understanding and controlling these flows. They also play a significant role in comparing analytical, numerical and experimental results as these results are for different scale flows depending on grid size and sensor size, etc. We develop these relations by examining flow response to multiplicative changes of spatial and temporal scales based on the scaling invariance of definitions of physical quantities. This reveals the self-similarity of flows, an invariance with respect to scaling like that appearing in many other fields, ${ }^{1-5}$ and shows the scaling invariance of fundamental laws governing the flows. We also derive both the necessary and sufficient condition for a physical quantity to be self-similar. In addition to their importance for fluid flows, the results are also valid for the other self-similar phenomena. ${ }^{1-5}$

Without losing generality, consider flows of incompressible, Newtonian fluids without the effect of external force fields. The governing equations are the continuity equation (conservation of mass) and Navier-Stokes equation (conservation of momentum):

$$
\begin{aligned}
& \operatorname{div} \mathbf{v}=0, \\
& \frac{\partial \mathbf{v}}{\partial t}+(\operatorname{grad} \mathbf{v}) \mathbf{v}=\nu \Delta \mathbf{v}-\operatorname{grad} p_{0} .
\end{aligned}
$$

The constitutive equation used in Eq. (2) is

$$
\mathbf{T}_{0}=-p_{0} \mathbf{I}+2 \nu \mathbf{D} .
$$

Here, $\mathbf{v}$ is the velocity vector, $p_{0}=p / \rho$ with $p$ as pressure and $\rho$ as density, $\nu$ is kinematical viscosity, $\mathbf{T}_{0}=\mathbf{T} / \rho$ with $\mathbf{T}$ as stress tensor, $\mathbf{I}$ is identity tensor, $\mathbf{D}$ is velocity strain tensor, $\Delta=$ div grad is the spatial Laplacian, and $t$ stands for time.

Consider a multiplicative change of spatial and temporal scales,

$$
\mathbf{x}^{*}=\sigma \mathbf{x}, \quad t^{*}=\sigma^{\alpha} t,
$$

${ }^{a)}$ Electronic mail: lqwang@hkucc.hku.hk where $\mathbf{x}$ stands for position vector, $\sigma$ is a scaling factor, and superscript $*$ denotes quantities at new scale $*$. Scaling index $\alpha$ relates to energy spectrum index $\beta$ in the form of ${ }^{1}$

$$
\beta=3-2 \alpha \text {. }
$$

$\beta$ is, for example, 3 for the flow in a buoyancy subrange between the containing eddy range and inertial range in a stable atmospheric boundary layer, ${ }^{6}$ and $5 / 3$ for the cascade process in Kolmogorov's turbulent range. ${ }^{7}$

Values of physical quantities such as $\mathbf{v}, p, \nu$, etc., usually vary upon the multiplicative change (4). This actually reflects different scale flows. However, their definitions are unchanged (e.g., the velocity $\mathbf{v}^{*}$ is still defined as the derivative of the position vector with respect to time at scale $*$ ). Such an invariance of physical definitions leads to

$$
\begin{aligned}
& \mathbf{v}\left(\sigma \mathbf{x}, \sigma^{\alpha} t\right)=\sigma^{1-\alpha} \mathbf{v}(\mathbf{x}, t), \quad \nu\left(\sigma \mathbf{x}, \sigma^{\alpha} t\right)=\sigma^{2-\alpha} \nu(\mathbf{x}, t), \\
& p_{0}\left(\sigma \mathbf{x}, \sigma^{\alpha} t\right)=\sigma^{2(1-\alpha)} p_{0}(\mathbf{x}, t), \\
& \mathbf{T}_{0}\left(\sigma \mathbf{x}, \sigma^{\alpha} t\right)=\sigma^{2(1-\alpha)} \mathbf{T}_{0}(\mathbf{x}, t), \\
& \mathbf{D}\left(\sigma \mathbf{x}, \sigma^{\alpha} t\right)=\sigma^{-\alpha} \mathbf{D}(\mathbf{x}, t),
\end{aligned}
$$

which show self-similarity of flows. Substituting Eq. (6) into Eqs. (1)-(3) yields,

$$
\begin{aligned}
& \operatorname{div}^{*} \mathbf{v}^{*}=0, \\
& \frac{\partial v^{*}}{\partial t^{*}}+\left(\operatorname{grad}^{*} \mathbf{v}^{*}\right) \mathbf{v}^{*}=\nu^{*} \Delta^{*} \mathbf{v}^{*}-\operatorname{grad}^{*} p_{0}^{*}, \\
& \mathbf{T}_{0}^{*}=-p_{0}^{*} \mathbf{I}+2 \nu^{*} \mathbf{D}^{*},
\end{aligned}
$$

which imply the scaling invariance of fundamental laws (1)(3). This partly confirms a fundamental conjecture that many laws of nature are independent, or nearly so, of a scaling factor. ${ }^{2,3}$

By the same argument, we can obtain

$$
\begin{aligned}
& \operatorname{Re}\left(\sigma \mathbf{x}, \sigma^{\alpha} t\right)=\operatorname{Re}(\mathbf{x}, t), \quad k\left(\sigma \mathbf{x}, \sigma^{\alpha} t\right)=\sigma^{2(1-\alpha)} k(\mathbf{x}, t), \\
& \varepsilon\left(\sigma \mathbf{x}, \sigma^{\alpha} t\right)=\sigma^{2-3 \alpha} \varepsilon(\mathbf{x}, t), \quad \omega\left(\sigma \mathbf{x}, \sigma^{\alpha} t\right)=\sigma^{-\alpha} \omega(\mathbf{x}, t), \\
& Q\left(\sigma \mathbf{x}, \sigma^{\alpha} t\right)=\sigma^{-2 \alpha} Q(\mathbf{x}, t), \\
& \varepsilon_{Q}\left(\sigma \mathbf{x}, \sigma^{\alpha} t\right)=\sigma^{-3 \alpha} \varepsilon_{Q}(\mathbf{x}, t), \\
& h\left(\sigma \mathbf{x}, \sigma^{\alpha} t\right)=\sigma^{1-2 \alpha} h(\mathbf{x}, t), \\
& \varepsilon_{h}\left(\sigma \mathbf{x}, \sigma^{\alpha} t\right)=\sigma^{1-3 \alpha} \varepsilon_{h}(\mathbf{x}, t),
\end{aligned}
$$


where Re is the Reynolds number, $k$ turbulent kinetic energy, $\varepsilon$ dissipation rate of turbulent kinetic energy, $\omega$ vorticity, $Q$ enstrophy, $\varepsilon_{Q}$ dissipation rate of turbulent enstrophy, $h$ helicity, and $\varepsilon_{h}$ dissipation rate of helicity. It is interesting to note that the Reynolds number is not only self-similar but also the scaling invariant for all flows. This is different from the other physical quantities, and contributes to its extremely important role in fluid mechanics.

The self-similar relations in Eqs. (6) and (10) may be regarded as special cases of a more general self-similar relation,

$$
f\left(\sigma \mathbf{x}, \sigma^{\alpha} t\right)=\sigma^{b} f(\mathbf{x}, t),
$$

where $f(\mathbf{x}, t)$ stands for a physical quantity, and $b$ is a function of $\alpha$ and different for different quantities. Note that $\sigma$ in Eq. (11) is essentially arbitrary except a limit imposed by Planck's constant and continuity assumption viewing fluid as a continuum (when things get too small) or the speed of light (when objects moves too fast). ${ }^{2,3}$ To obtain a necessary condition, taking $\sigma=t^{-1 / \alpha}$ yields

$$
f(\mathbf{x}, t)=t^{b / \alpha} G\left(t^{-1 / \alpha} \mathbf{x}\right),
$$

where $G\left(t^{-1 / \alpha} \mathbf{x}\right)=f\left(t^{-1 / \alpha} \mathbf{x}, 1\right)$. If Eq. (12) holds,

$$
\begin{aligned}
f\left(\sigma \mathbf{x}, \sigma^{\alpha} t\right) & =\left(\sigma^{\alpha} t\right)^{b / \alpha} G\left[\left(\sigma^{\alpha} t\right)^{-1 / \alpha} \sigma \mathbf{x}\right] \\
& =\sigma^{b} t^{b / \alpha} G\left(t^{-1 / \alpha} \mathbf{x}\right)=\sigma^{b} f(\mathbf{x}, t),
\end{aligned}
$$

which establishes the sufficiency of Eq. (12) for Eq. (11).

Considering the cascade process in Kolmogorov's turbu- lent range with $\beta=5 / 3$ and $\alpha=2 / 3$ [Eq. (5)], Eq. (12) leads to an expression for velocity field,

$$
\mathbf{v}(\mathbf{x}, t)=t^{1 / 2} G\left(t^{-3 / 2} \mathbf{x}\right) .
$$

For this flow, the dissipation rate $\varepsilon$ of turbulent kinetic energy is independent of scaling because $\alpha=2 / 3$ [Eq. (10)].

The necessary and sufficient condition for self-similar quantities [Eq. (12)] is also valid for other self-similar phenomena.

Financial support from the Research Grants Council of Hong Kong and the Committee on Research and Conference Grants of the University of Hong Kong is gratefully acknowledged.

${ }^{1}$ H. O. Peitgen, H. Jürgens, and D. Saupe, Chaos and Fractals: New Frontiers of Science (Springer, Berlin, 1992).

${ }^{2}$ M. R. Schroeder, Fractals, Chaos, Power Laws (Freeman, New York, 1991).

${ }^{3}$ M. R. Schroeder, Number Theory in Science and Communication with Applications in Cryptography, Physics, Digital Information, Computing, and Self-similarity, 3rd ed. (Springer, Berlin, 1997).

${ }^{4}$ R. V. Sole, S. C. Manrubia, M. Benton and P. Bak, Nature (London) 388, 764 (1997).

${ }^{5}$ H. E. Stanley, L. A. N. Amaral, S. V. Buldyrev, A. L. Goldberger, S. Havlin, H. Leschhorn, P. Maass, H. A. Makse, C. K. Peng, M. A. Salinger, M. H. R. Stanley, and G. M. Viswanathan, Physica A 231, 20 (1996).

${ }^{6}$ R. B. Stull, An Introduction to Boundary Layer Meteorology (Kluwer, Dordrecht, 1988).

${ }^{7}$ U. Frisch, Turbulence: The Legacy of A. N. Kolmogorov (Cambridge University Press, Cambridge, 1995). 